\title{
Silicon Electrochemical Etching for Sensors and Electron Devices Fabrication
}

\author{
Shinobu Suwazono, Keizo Yamada* and Toshihide KURIYama
}

Received July 31, 1991; Accepted September 17, 1991

\begin{abstract}
Electrochemical etch-stop technology using hydrazine hydrate has been developed for fabrication of silicon mechanical sensors, such as pressure sensors and accelerometers, and silicon on insulator structure for electron devices. Etch-stop conditions for $n$ type silicon and $p$ type silicon were obtained by the three electrodes method, using a potentiostat. By applying a four electrodes method for etch-stop, selective etching for $p$ type silicon substrate was achieved. When an $n$ type epitaxial silicon layer with $p$ type silicon substrate was used, thin silicon diaphragm structure for mechanical sensors was obtained by selective etching of $p$ type silicon substrate. After the $\mathbf{n}$ type epitaxial silicon layer with $\mathbf{p}$ type silicon substrate was directly bonded with the other silicon wafer covered with silicon dioxide, a silicon on insulator structure was also obtained by selectively etching the $p$ type substrate.

Thicknesses for diaphragm and insulated layer were well controlled, for example $19.5 \pm 0.5 \mu \mathrm{m}$.
\end{abstract}

\section{INTRODUCTION}

In silicon devices, thickness control is very important for device fabrication. For example, in silicon mechanical sensors, such as pressure sensors and accelerometers, thickness of diaphragm and beam directly influence their sensitivity. In electron devices using silicon on insulator (SOI) substrate, a thin silicon layer is suitable for lateral device isolation.

There have been many etch-stop methods for controlling silicon thickness. Recently, electrochemical etch-stop was reported for thickness control of silicon membranes ${ }^{1-3)}$. In the method, $p$ type silicon with a thin $n$ type epitaxial silicon layer was used and the $p$ type silicon was selectively etched away. By using the four electrode

Sensor Research Laboratory, Microelectronics Research Laboratories. NEC Corporation (1120, Shimokuzawa, Sagamihara, Kanagawa 229, Japan)

Key Words: Sensor, Electrochemical Etching, SOI, Silicon, Hydrazine, Pressure, Accelerometer method $^{3)}$, electrical potentials for $\mathrm{p}$ type silicon, $n$ type silicon and $\mathrm{KOH}$ etching solution were fixed using a reference electrode and a potentiostat. In this work, electrochemical etching using hydrazine hydrate was used for silicon membrane thickness control and the method was applied for sensors and SOI structure fabrication. Hydrazine hydrate does not contain alkaline ions, making it suitable for electron device formation, using the thin silicon layers.

\section{EXPERIMENTAL}

First, electrochemical etch stop conditions were checked for both $\mathrm{n}$ type silicon and $\mathrm{p}$ type silicon. In $p$ type silicon, it was easy to make a contact with metal. In $\mathrm{n}$ type silicon, on the other hand, it was difficult to make a good ohmic contact with metal. To get a good contact, aluminum was partially deposited on both silicon wafers. Figure 1 shows the I-V characteristics, when the silicon potential was changed. A $\mathrm{Ag} / \mathrm{AgCl}$ reference electrode (Toa denpa Kogyo 


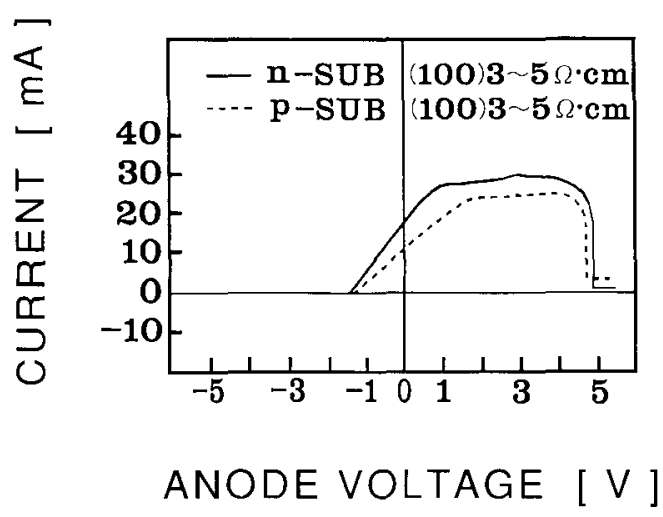

Fig. 1 I.V characteristics for $p$ type silicon and $n$ type silicon.

HS-605C) was used for hydrazine hydrate potential measurement. The silicon potential was supplied by a potentiostat (Hokuto Denko Ltd. HA-501). As the silicon potential increased, current increased until it suddenly decreased where etching stopped by anodic oxidation of the silicon surface.

Silicon diaphragm formation for pressure sensors was accomplished by using 2 inch diameter $40 \Omega \cdot \mathrm{cm}$ p type silicon substrate with 20 $\mu \mathrm{m}$ thick $5 \Omega \cdot \mathrm{cm} \mathrm{n}$ type silicon epitaxial layer. The silicon wafer was thermally oxidized. The silicon dioxide was $3000 \AA$ thick and was used as an hydrazine etching mask. It was then etched by buffered hydrofluoric acid (HF) to make an etching pattern. Al electrodes was made on a part of the $p$ type silicon and $n$ type silicon surface. Figure 2 shows an experimental setup for electrochemical etching. $\mathrm{N}$ type silicon and $\mathrm{p}$ type silicon layers were connected to working electrodes of dual potentiostat (Hokuto Denko Ltd), respectively. $\mathrm{N}$ type silicon potential was set at $9 \mathrm{~V}$ and $\mathrm{p}$ type silicon potential was set at $-1.4 \mathrm{~V}$ (rest potential). A special wafer mount was used to protect the wafer periphery from hydrazine hydrate solution, stopping current leakage at the wafer edge. The leak current had a large influence on the etching condition. Although the influence could be greatly reduced by using the four electrode method, it was still important to decrease the leak current to fix a potential distribution in the wafer. The

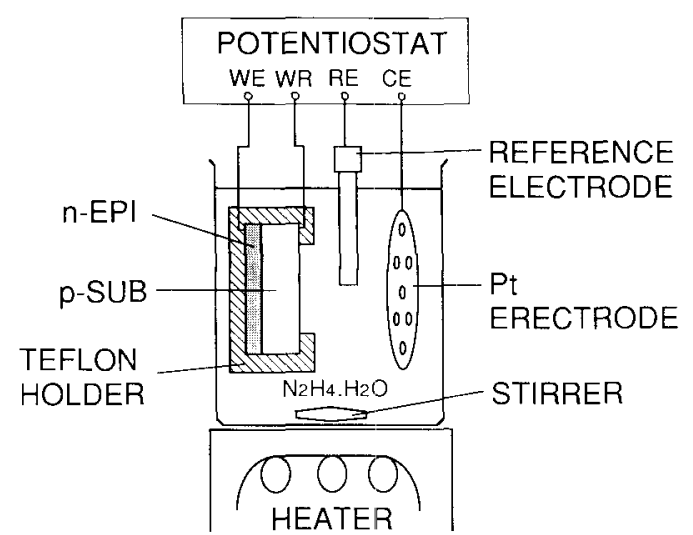

Fig. 2 Experimental setup for electrochemical etching.

hydrazine hydrate solution was stirred and kept $80^{\circ} \mathrm{C}$.

\section{RESULTS AND DISCUSSION}

Figure 3 shows the current values at the two working electrodes during etching. In this case, the p-n junction had a current leak of $10 \mathrm{~mA}$. When the $p$ type substrate was etched away and the $\mathrm{n}$ type layer appeared, an anode current increased, then decreased. After the current came back to the original value, the wafer was taken out from the solution and washed with deionized water. The diaphragm thickness was measured by a microscope, after the wafer was cleaved. The value was $26 \pm 0.5 \mu \mathrm{m}$. Thickness difference between the $\mathrm{n}$ type silicon and the diaphragm is considered to be due to a depletion layer at a p-njunction, which will be discussed.

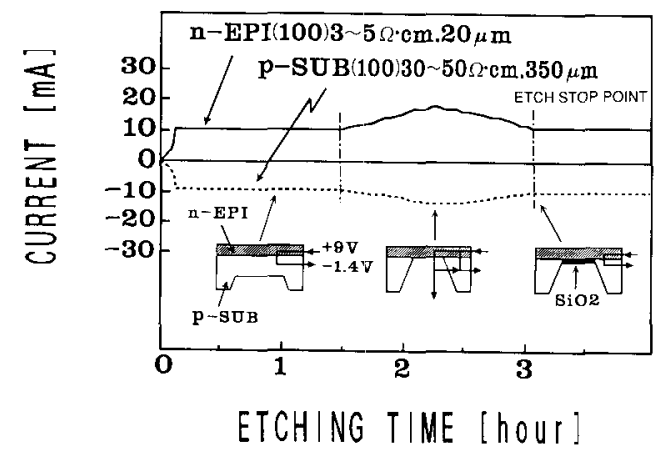

Fig. 3 Current values at $n$ type silicon and $\mathrm{p}$ type silicon. 
In the case of an accelerometer, the $p$ type silicon substrate should remain in island shape in the membrane, to form a weight for acceleration detection. When a silicon wafer with (100) surface is used, the side wall surface is (111) and etching is also stopped at the pattern size. The silicon island, however, has corners and etching does not stop at the corner, because the (211) surface appears at the corner and the surface was etched by hydrazine hydrate. To get a rectangular silicon weight in the center membrane, a correcting pattern is necessary. In this case, another small square is added at each corner of the weight. As etching proceeds, the small square is etched off and disappears. $400 \mu \mathrm{m}$ square, with its center positioned at the weight corner, was used for correction the pattern, as shown in Fig. 4. Figure 5 shows the current for the two working electrodes. In this wafer, current leak between $n$ type silicon and $p$ type silicon was very small. As the $p$ type silicon was etched away, current flows from $n$ type silicon to hydrazine solution and a small current flows from the $\mathrm{n}$ type silicon to the $\mathrm{p}$ type silicon. In the case of an accelerometer, corners of the silicon island for weight continued to be etched, and the current did not reach a constant value. Etching was finished and a wafer was removed from the hydrazine solution, when the current reached a minimum value which is shown Fig. 5 by an arrow. If the etching was continued, the current began to increase because the corner of silicon island continued to be etched and an additional $n$ type silicon area appeared. When a silicon wafer with $15 \mu \mathrm{m}$ thick $\mathrm{n}$ type silicon, the diaphragm thickness was $19.5 \pm 0.5 \mu \mathrm{m}$. The corners of the weight were well etched and the shape was almost rectangular.

In electron devices such as MIS FET ICs (Metal Insulator Semiconductor Field Effect Transistor Integrated Circuits), SOI (Silicon on Insulator) structure has been paid attention because of its excellent dielectric isolation properties. This structure were applied to such electron devices as alpha particle resistant ICs, high speed ICs and high voltage ICs. Many kinds of SOI fabrication

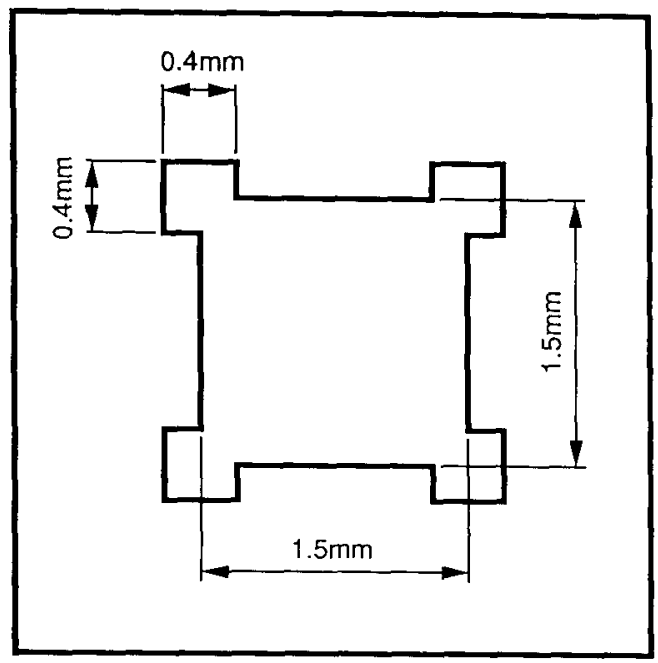

Fig. 4 Correcting pattern for an accelerometer weight.

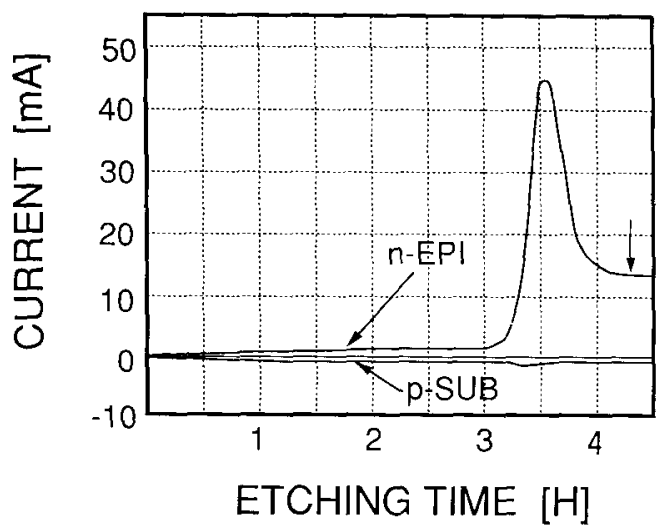

Fig. 5 Current values at $n$ type silicon and $p$ type silicon.

methods were reported. Recently, a silicon to silicon direct bonding method has become a strong candidate for SOI structure fabrication ${ }^{4}$. In the silicon to silicon direct bonding method, silicon wafers covered with silicon dioxide are bonded without any other adhesive material. Only heating in a nitrogen atmosphere causes good adhesion between the two wafers. To obtain a thin silicon layer on the insulating layer, it is necessary to eliminate almost all the silicon on one side. This has conventionally been done by grinding and polishing one of the silicon wafer pair. This method, however, involved a difficulty in controlling the silicon layer thickness, because 
only a 1 to $20 \mu \mathrm{m}$ thick silicon layer should remain, in spite of the fact that the total thickness for the pair silicon wafer is almost $1 \mathrm{~mm}$. To solve this problem, some chemical etching methods had been proposed. These methods still involved difficulty in obtaining a thin uniform layer. The authors applied the above mentioned electrochemical etching to obtain a SOI structure. The advantage, in regard to hydrazine hydrate used for such devices, is that it does not contain alkaline ions. Alkaline ions influence unfavourably on the transistor characteristics.

In this paper, after a $\mathrm{p}$ type silicon wafer with $15 \mu \mathrm{m}$ thick $\mathrm{n}$ type silicon epitaxial layer was oxidized to have $1000 \AA$ thick silicon dioxide, it was directly bonded to the other silicon wafer with a $500 \AA$ thick silicon dioxide. To fix the potential of the etched silicon wafer, ring shape $\mathrm{n}^{+}$region were made at the edge of the wafer. The etching procedure was the same as described above.

Figure 6 shows a cross sectional structure of the directly bonded wafers before the electrochemical etching (a) and a photograph of SOI wafer obtained by the electrochemical etching (b). Figure 7 shows the current for an $\mathrm{n}$ type silicon Almost the same current shape was observed and good thickness control was obtained. Figure 8 shows the thickness distribution in the wafer. The thickness was measured by a microscope after the wafer was cut.

As shown in the above experiments, it is very important to fix the electrical potential of both $n$ type silicon and $p$ type silicon in the case of electrochemical etch stop. When leak current between $n$ type silicon and $\mathrm{p}$ type silicoñ was small, it was possible to use a three electrode method where $\mathrm{p}$ type silicon was not connected to any electrodes. This phenomenon can be explained by the fact that a $p$ type silicon potential is also influenced by the hydrazine hydrate potential in the case of a three electrode method. When the conductance between the $p$ type silicon and the hydrazine hydrate is much larger than the conductance between the $p$ type (a)

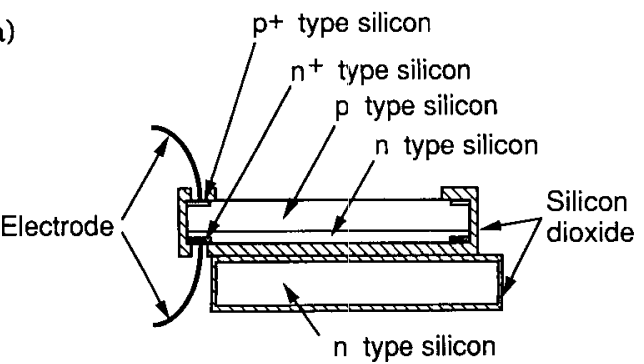

(b)

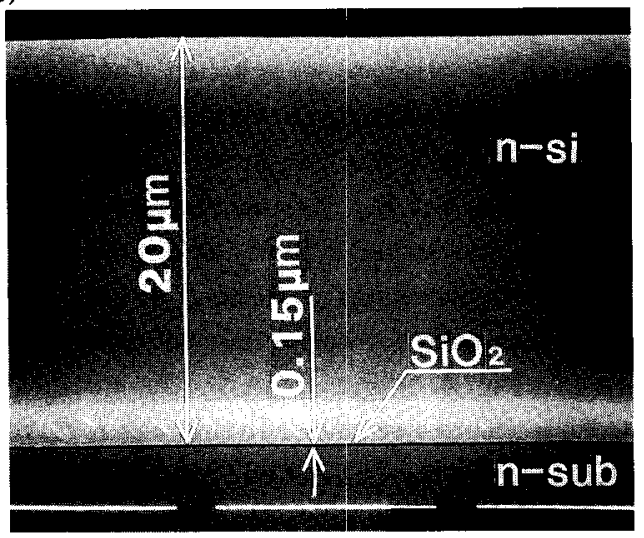

Fig. 6 Cross sectional structure of directly bonded wafers before etching (a) and a photograph of SOI wafer (b).

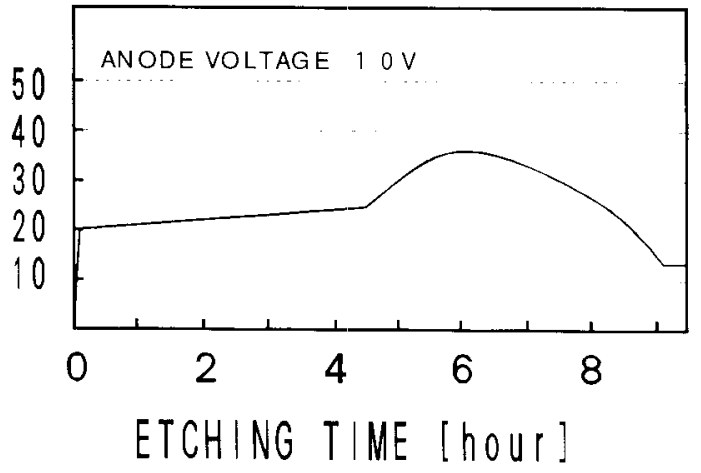

Fig. 7 Current values at $n$ type silicon.

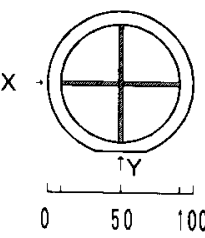

WAFER SIZE [mm]

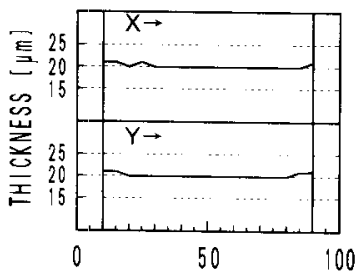

WAFER MEASUREMENT POINT [mm]
Fig. 8 Thickness distribution in the SOI wafer. 
silicon and the $\mathrm{n}$ type silicon, the $\mathrm{p}$ type silicon potential is fixed to nearly the same potential with the hydrazine hydrate and the $p$ type silicon is etched away.

In the case of the hydrazine hydrate etching, an etch stop position does not locate at p-n junction. The diaphragm thickness was equal to sum of the epitaxial layer thickness and the depletion region thickness. Etching is considered to stop at the edge of a depletion region in the $p$ type silicon.

In the case of a $\mathrm{KOH}$ etching, it was reported that an etch stop position locates at a $p-n$ junction ${ }^{3}$. The difference of the etch stop position is considered to be due to an etching rate difference of silicon dioxide and an etch stop potential difference between hydrazine hydrate and $\mathrm{KOH}$. The etching rate of silicon dioxide is much smaller in hydrazine hydrate than in $\mathrm{KOH}$. A very thin silicon dioxide produced by anodic oxidation can effectively stop etching in the case of hydrazine hydrate. The etch stop potentials of silicon were about $5 \mathrm{~V}$ in the case of hydrazine hydrate and about $-1 \mathrm{~V}$ in the case of $\mathrm{KOH}$. A higher potential is necessary in the hydrazine hydrate etch stop, which makes the depletion region of the p-n junction wider ${ }^{2}$.

\section{CONCLUSION}

Electrochemical etch-stop technology using hydrazine hydrate was developed to obtain a thin $n$ type silicon layer. The thin silicon layer will be effectively applied to a diaphragm formation and a beam formation in the case of mechanical sensors, such as a pressure sensor and an accelerometer. An SOI structure was also successfully obtained by combining the electrochemical etching and silicon direct bonding. Hydrazine hydrate does not contain alkaline ions and is suitable for etching of silicon for electron devices fabrication.

The authors would like to thank Dr. K. Higuchi and Mr. H. Tanigawa for their encouragement and useful discussions. Thanks are also due to Dr. J. Kimura for his advice.

\section{REFERENCES}

1) S. C. Kim and K. D. Wise, IEEE Transactions on Electron Devices, ED-30, 802, (1983).

2) M. Hirata, S. Suwazono and H. Tanigawa, Electrochem. Soc., 134, 2037, (1987).

3) B. Kloeck, S. D. Collins, N. F. de Rooij and R. L. Smith, IEEE Transactions on Electron Devices, 36, $663(1989)$.

4) H. Ohasi, J. Ohura, T. Tsukakoshi and M. Simbo, Proceedings of IEDM, p.210(1986). 